\title{
Barriers and facilitators to HPV vaccination in primary care practices: a mixed methods study using the Consolidated Framework for Implementation Research
}

\author{
Jane M. Garbutt ${ }^{1 *}$, Sherry Dodd ${ }^{2}$, Emily Walling ${ }^{2,3}$, Amanda A. Lee ${ }^{4}$, Katharine Kulka ${ }^{2}$ and Rebecca Lobb ${ }^{4}$
}

\begin{abstract}
Background: In the United States, the effective, safe huma papilloma virus (HPV) vaccine is underused and opportunities to prevent cancer continue to be missed. National guidelines recommend completing the 2-3 dose HPV vaccine series by age 13, well before exposure to the sexually transmitted virus. Accurate characterization of the facilitators and barriers to full implementation of HPV vaccine recommendations in the primary care setting could inform effective implementation strategies.
\end{abstract}

Methods: We used the Consolidated Framework for Implementation Research (CFIR) to systematically investigate and characterize factors that influence HPV vaccine use in 10 primary care practices (16 providers) using a concurrent mixed methods design. The CFIR was used to guide collection and analysis of qualitative data collected through in-person semi-structured interviews with the primary care providers. We analyzed HPV vaccine use with data abstracted from medical charts. Constructs that most strongly influenced vaccine use were identified by integrating the qualitative and quantitative data.

Results: Of the 72 CFIR constructs assessed, seven strongly distinguished and seven weakly distinguished between providers with higher versus lower HPV vaccine coverage. The majority of strongly distinguishing constructs were facilitators and were related to characteristics of the providers (knowledge and beliefs; self-efficacy; readiness for change), their perception of the intervention (relative advantage of vaccinating younger vs. older adolescents), and their process to deliver the vaccine (executing). Additional weakly distinguishing constructs that were facilitators were from outer setting (peer pressure; financial incentives), inner setting (networks and communications and readiness for implementation) and process (planning; engaging, and reflecting and evaluating). Two strongly distinguishing constructs were barriers to use, one from the intervention (adaptability of the age of initiation) and the other from outer setting (patient needs and resources).

Conclusions: Using CFIR to systematically examine the use of this vaccine in independent primary care practices enabled us to identify facilitators and barriers at the provider, interpersonal and practice level that need to be addressed in future efforts to increase vaccine use in such settings. Our findings suggest that implementation strategies that target the provider and help them to address multi-level barriers to HPV vaccine use merit further investigation.

Keywords: HPV vaccine, Implementation strategies, CFIR

\footnotetext{
* Correspondence: jgarbutt@wustl.edu

${ }^{1}$ Departments of Medicine and Pediatrics, Washington University School of

Medicine, St. Louis, MO, USA

Full list of author information is available at the end of the article
}

(c) The Author(s). 2018 Open Access This article is distributed under the terms of the Creative Commons Attribution 4.0 International License (http://creativecommons.org/licenses/by/4.0/), which permits unrestricted use, distribution, and reproduction in any medium, provided you give appropriate credit to the original author(s) and the source, provide a link to the Creative Commons license, and indicate if changes were made. The Creative Commons Public Domain Dedication waiver (http://creativecommons.org/publicdomain/zero/1.0/) applies to the data made available in this article, unless otherwise stated. 


\section{Background}

A vaccine targeting oncogenic human papilloma viruses (HPV) is an important health innovation. The HPV vaccine is predicted to prevent $\sim 70 \%$ of cervical cancers as well as other genitourinary and oral cancers and genital warts in men and women. [1,2] The vaccine has been recommended for routine use in the United States for over ten years [1] and already has been shown to prevent up to $99 \%$ of HPV-related cervical dysplasia, a precursor of cervical cancer. [1-3] However, the full benefit of the HPV vaccine can only be realized if the vaccination series is completed prior to exposure to the sexually transmitted virus. National guidelines recommend giving the vaccine to all adolescents at their 11 to 12 year old check-up visit concurrently with the tetanus, diphtheria, pertussis booster (Tdap) and the meningococcal conjugate vaccine (MCV4), and completing the HPV vaccine series before their 13th birthday. [4] Until a few months ago, the recommended series comprised 3 doses given over 6 months. At the end of 2016, this changed to 2 doses of the 9-valent vaccine given over 6 to 12 months, if the series is initiated before the 15 th birthday. [5]

Introduced in the United States (U.S.) for girls in 2006 and for boys in 2011, use of this effective vaccine is far below the Healthy People 2020 objective of $80 \%$ coverage by age 13 to 15 for both sexes. [6] In 2015, only 41 . $9 \%$ of eligible females and $28.1 \%$ of eligible males aged 13-17 years had completed the 3-dose series, with marked variation among states. [7] Opportunities to prevent cancer continue to be missed. There is an obvious disparity in uptake of HPV vaccine when compared with coverage for Tdap (86.4\%) and MCV4 (81.3\%) in the same age-group. [7] Interventions to increase initiation of the HPV vaccine and series completion targeting both parents and providers that have been rigorously evaluated, have had at best a modest effect ( $\sim$ increase). [1, 8]' [9] Many studies showing larger effects have been criticized for being methodologically deficient. $[1,9,10]$ Alternative approaches to increase use of HPV vaccine are urgently needed. Accurate characterization of the facilitators and barriers to full implementation of HPV vaccine recommendations in the primary care setting could inform effective implementation strategies.

Closing the gap between the national goal and current vaccine use requires improved uptake in primary care settings where the majority of children are vaccinated. We hypothesized that in these settings, factors that influence HPV vaccine use are multifactorial and complex. The objective of this study was to use a systematic approach based on the Consolidated Framework for Implementation Research (CFIR) [11] to identify and characterize factors that are facilitators of and barriers to implementation of the national recommendations for HPV vaccine use. We will use this information to inform development of a pragmatic and effective implementation strategy to increase timely use of this important oncogenic vaccine.

\section{Methods}

We assessed the implementation of HPV vaccine in ten community pediatric offices using a concurrent mixed methods design. Quantitative and qualitative data were analyzed separately and then were integrated to provide additional information. [12] Specifically, we conducted a retrospective analysis of HPV vaccine use data abstracted from medical charts and acquired during in-person semi-structured interviews with the pediatric primary care providers. The study was approved by the Washington University Human Research Protection Office.

The CFIR was used to guide development of the interview guide, data coding, and data analysis. Damschroder and colleagues developed the CFIR to consolidate and unify key constructs from 19 published implementation theories. [11] Seventy-two constructs are grouped under five major domains that influence successful implementation of evidence-based interventions. These include characteristics of the intervention (in this case, use of $\mathrm{HPV}$ vaccine in accordance with CDC guidelines), the inner setting (the pediatric practice), the outer setting (the context in which the pediatric practice resides), individual characteristics of the implementers (the healthcare providers), and the processes used for implementation of the intervention. [11] Definitions of the constructs within each domain can be found at 13012 2008_182_MOESM3_ESM.pdf [11].

\section{Study participants}

We invited all members of the Washington University Pediatric and Adolescent Ambulatory Research Consortium (WU PAARC) to participate. WU PAARC is a practicebased research network of 79 community pediatricians and 6 pediatric nurse practitioners associated with Washington University. A convenience sample of 16 providers from ten practices volunteered to participate. Each participant provided written consent and received $\$ 50$ as a token of appreciation for their time.

\section{Advisory board}

The study was guided by an Advisory Board of three pediatricians, one pediatric nurse practitioner, and two parents. The three pediatricians volunteered to be study subjects. The group met five times over the 12-month study period to review materials, problem solve, and assist in interpretation of study findings. 


\section{Data collection \\ Quantitative data}

For each provider, we abstracted data on vaccine coverage from medical charts for girls and boys who were 11 to 15 years old and had attended at least one office visit between January 1st, 2014 and December 31st, 2014. Data abstracted from the chart included: gender, date of birth, date of vaccine and type of vaccine (HPV, Tdap and MCV4).

\section{Qualitative data}

We developed an interview guide with a series of openended questions and probes to elicit examples that would clarify and illustrate the participant's responses. The guide included general and specific questions that addressed the main CFIR constructs to determine those that supported or created barriers to implementation. We explored how each of these constructs differed from that used for other adolescent vaccines to gather information to explain the higher coverage for the other childhood vaccines. The interviews were conducted between $1 / 27 / 2016$ and $5 / 24 / 2016$, by the principal investigator (JG) and co-investigator (EW). Interviews were designed to last about $30 \mathrm{~min}$ and were conducted at the practices. All were digitally recorded and transcribed verbatim by trained transcriptionists. After the qualitative interview, participants completed a short, structured survey describing their practice arrangements.

\section{Data analysis}

\section{Quantitative data}

For each practice and provider, we computed the overall coverage with HPV vaccine as the percentage of girls and boys who received three doses by their 13th birthday (a HEDIS measure for adolescent care). [13]

\section{Qualitative data}

We used a deductive approach to coding guided by the CFIR domains and constructs. We were also open to new themes that emerged inductively from the data. The transcripts were organized using NVivo software and each transcript was analyzed using consensual qualitative research methods with multiple analysts from diverse disciplines (e.g. pediatrician, implementation scientist, health services researcher) to foster multiple perspectives and validation. [14] We used an iterative process to develop a codebook that reflected each CFIR domain and its constructs, and key themes that emerged by refining the code definitions through consensus. The coding process began with two analysts who independently reviewed each transcript to identify the text units that were associated with each of the CFIR domains. Subsequently, they compared assessments, discussed differences and came to agreement on final text assignments. The text within each CFIR domain was further coded by three analysts to identify constructs within each CFIR domain. Thus, each transcript was subjected to a two-level consensus process.

\section{Rating the CFIR constructs}

Each transcript was subjected to a rating process. Five analysts used a deliberated consensus process to assign a rating to each construct within each transcript. The ratings reflected the valence (positive or negative influence) of the construct on implementation of HPV vaccine according to national guidelines (3 doses completed by the 13th birthday). Table 1 provides the criteria used to guide the rating process.

\section{Integrating the rating of CFIR constructs and coverage rates} Next, for each provider, we integrated their construct ratings with their HPV vaccine coverage in a table (An example is provided in Table 2). We grouped physicians by coverage rates using tertiles: lower coverage $<5 \%$ ( $n$ $=5$ providers $)$, moderate coverage $6-19 \%(n=6)$, and higher coverage $>20 \%(n=5)$. This approach allowed us to observe patterns of ratings for CFIR domains and constructs among lower and higher coverage providers and generate hypotheses about constructs that distinguished between groups. We focused on extreme values of coverage to ascertain the most information. [14] Strongly and weakly distinguishing patterns were determined based on two dimensions within and across higher and lower coverage groups. We first assessed the pattern of positive and negative ratings for each construct within coverage groups (higher and lower) to determine if the factor was a facilitator or barrier to use of HPV vaccine. Next, we examined the difference in

Table 1 Criteria Used to assign Ratings to Constructs

\begin{tabular}{ll}
\hline Rating & Criteria \\
\hline-1 & $\begin{array}{l}\text { A negative influence on providing HPV vaccine according to guideline recommendations i.e., complete the 3-dose series by the child's 13th } \\
\text { birthday. The interviewee provides examples of how key aspects of the construct exert a negative impact on HPV vaccine use, reports an } \\
\text { overall negative effect, or sufficient information is provided to make indirect inference about a negative effect. }\end{array}$ \\
& Appears to have no effect on use of HPV vaccine according to guideline recommendations. \\
+1 & $\begin{array}{l}\text { A positive influence on providing HPV vaccine according to guideline recommendations i.e., complete the 3-dose series by the child's 13th } \\
\text { birthday. The interviewee provides examples of how key aspects of the construct exert a positive impact on HPV vaccine use, reports an } \\
\text { overall positive effect, or sufficient information is provided to make indirect inference about a positive effect. }\end{array}$ \\
\hline
\end{tabular}


Table 2 An example of the table of integrated data from qualitative and quantitative studies comparing ratings for CFIR constructs in the characteristics of individuals domain and HPV vaccine coverage data (completion of 3 doses by 13th birthday)

\begin{tabular}{|c|c|c|c|c|c|c|c|c|c|c|c|c|c|c|c|c|}
\hline CHARACTERISTICS OF INDIVIDUALS & 1013 & 1004 & 1007 & 1015 & 1005 & 1001 & 1009 & 1020 & 1019 & 1008 & 1014 & 1018 & 1010 & 1006 & 1011 & 1003 \\
\hline HPV vaccine coverage & $25.5 \%$ & $24.6 \%$ & $24.0 \%$ & $23.0 \%$ & $20.5 \%$ & $18.1 \%$ & $16.5 \%$ & $16.1 \%$ & $8.7 \%$ & $8.1 \%$ & $6.1 \%$ & $4.8 \%$ & $4.6 \%$ & $4.2 \%$ & $3.7 \%$ & $1.2 \%$ \\
\hline \multicolumn{17}{|l|}{ Knowledge and Beliefs } \\
\hline $\begin{array}{l}\text { Value completing the } \\
\text { vaccine by age } 13\end{array}$ & + & + & + & + & + & + & + & - & + & + & + & - & - & - & + & - \\
\hline Believes benefit in bundling & + & + & + & + & + & + & + & - & + & + & - & + & + & - & + & - \\
\hline \multicolumn{17}{|l|}{ Self-Efficacy } \\
\hline Enthusiastic use of HPV vaccine & + & + & + & + & + & + & + & + & + & + & + & + & + & - & + & - \\
\hline $\begin{array}{l}\text { Familiarity with facts about HPV } \\
\text { vaccine }\end{array}$ & + & + & + & + & + & + & + & + & + & + & + & + & + & - & + & + \\
\hline $\begin{array}{l}\text { Confident to recommend } \\
\text { strongly at early age }(11-12)\end{array}$ & + & + & + & + & + & + & + & + & + & + & + & - & - & - & + & - \\
\hline $\begin{array}{l}\text { Confident in ability to convince } \\
\text { hesitant parents }\end{array}$ & + & - & + & + & + & - & + & + & + & + & - & - & - & - & + & - \\
\hline Readiness to change & + & - & + & + & + & - & + & - & - & - & + & - & - & - & + & - \\
\hline
\end{tabular}

positive or negative ratings in the higher and lower coverage groups to determine if the construct was an important distinguishing factor. If this difference was $\geq$ $60 \%$ (e.g., $5 / 5$ or $100 \%$ positive in higher coverage and $1 / 5$ or $20 \%$ positive in lower coverage), the construct was considered to be "strongly distinguishing." If this difference was between $40 \%$ to $59 \%$ (e.g., $5 / 5$ or $100 \%$ positive in higher coverage and $3 / 5$ or $60 \%$ positive in lower coverage), the construct was considered to be "weakly distinguishing." If the difference was $<40 \%$ (e.g. , $5 / 5$ or $100 \%$ positive in higher coverage and $4 / 5$ or $80 \%$ positive in lower coverage), the construct was determined to provide limited information about coverage rates. To support the validity of our conclusions, we checked with members of the Advisory Board. [14]

\section{Results}

Sixteen providers participated in both the quantitative and qualitative studies. They comprised 15 pediatricians and one pediatric nurse practitioner. Five were male, 11 were female, 13 were Caucasian, 2 were African American, and one was Asian. The 16 providers were drawn from ten practices, nine of which had multiple providers (2-6 providers per practice). Two practices were part of a healthcare group and had access to central support for information systems and quality improvement staff. Such resources were not available at other practices. Eight practices used an electronic medical record (EMR).

\section{Quantitative data}

Medical records from a total of 4592 teens were eligible for inclusion in the data analysis of coverage rates. Overall, $13.9 \%$ of eligible teens had received 3-doses of HPV vaccine before their 13 th birthday (16.9\% girls, $11.3 \%$ boys). This metric varied among practices from $2.3 \%$ to $25.5 \%$ and there was wide variability in HPV vaccine coverage among providers within the same practice (Table 3). Consequently, we chose to analyze the qualitative data at the level of the individual provider rather than at the level of the practice.

\section{Qualitative data}

CFIR constructs are not reported if we found limited text that could be coded within that domain construct. For intervention domain, this included the following constructs: intervention source, evidence strength and quality, trialability, design quality and packaging. For the inner setting domain, this included: structural characteristics, culture, compatibility, organizational incentives and rewards, and learning climate. For the characteristics of individuals domain, we found no text about individual identification with the organization. Within the process domain, we did not identify any opinion leaders, internal implementation leaders or champions.

\section{Innovation characteristics domain Relative advantage}

The most commonly mentioned perceived advantages to providing HPV vaccine at age 11-12 years rather than to older teens were increased immunogenicity, successfully completing the series before exposure to HPV and accessing the child at their 11-12 year old check-up required by school. Although the vaccine can be given to children as young as 9 , no providers did so routinely. Several providers offered it to younger children accompanying an older sibling to a check-up visit where they received $\mathrm{HPV}$ vaccine. 
Table 3 Coverage Rates for HPV Vaccine by Practice and Provider

\begin{tabular}{|c|c|c|c|c|}
\hline \multirow[t]{2}{*}{ Practice } & \multirow[t]{2}{*}{ Number of children age 13} & \multirow[t]{2}{*}{ Electronic medical record (EMR) or paper records } & \multicolumn{2}{|c|}{ Completed 3 doses HPV by 13 th birthday } \\
\hline & & & Practice coverage & Provider coverage \\
\hline A & 329 & EMR & $25.5 \%$ & $25.5 \%$ \\
\hline B & 383 & EMR & $23.0 \%$ & $23.0 \%$ \\
\hline$C^{a}$ & 299 & EMR & $18.1 \%$ & $18.1 \%$ \\
\hline D & 726 & EMR & $18.6 \%$ & $24.0 \%, 3.7 \%$ \\
\hline$E$ & 441 & Paper records & $13.8 \%$ & $24.6 \%, 16.1 \%, 4.2 \%$ \\
\hline$F^{a}$ & 940 & EMR & $10.6 \%$ & $20.5 \%, 4.6 \%$ \\
\hline G & 636 & EMR & $9.6 \%$ & $16.5 \%, 8.1 \%$ \\
\hline $\mathrm{H}$ & 416 & EMR & $8.7 \%$ & $8.7 \%$ \\
\hline | & 293 & EMR & $6.1 \%$ & $6.1 \%$ \\
\hline J & 129 & Paper records & $2.3 \%$ & $4.8 \%, 1.2 \%$ \\
\hline All & 4592 & & $13.9 \%$ & \\
\hline
\end{tabular}

Practice was part of a healthcare group with access to information system assistance and quality improvement staff

\section{Adaptability}

All providers adapted the vaccine dosing schedule, most often to provide the 2nd and 3rd doses at subsequent annual checkup visits. Some providers also delayed the age of initiation beyond 11-12 years to prevent teens from having three vaccines concurrently or to avoid conflict with a family they thought might be uncomfortable discussing prevention of a sexually transmitted disease at this age.

\section{Complexity}

The majority of providers identified at least one difficulty with administering the 3-dose series. Many cited the resistance and hesitancy of parents, as exemplified by this quote:

"Well, I think you have to go in more proactively with that one [HPV], because you expect a fight. You do, because nine times out of ten, you have a parent who has heard something about it who has a problem with it. You are trying to push the vaccine in a positive way before the negative parts come in from the parent."

Others mentioned that it took longer to discuss HPV vaccine than other adolescent vaccines and less frequent visits of older adolescents increased the difficulty of completing the series.

\section{Cost}

Few providers mentioned cost associated with providing HPV vaccine, although several noted that providing the 3 -dose series was revenue generating.

\section{Outer setting domain}

\section{Patient needs and resources}

Most providers felt it was important to provide information about vaccine benefits as reported by this provider:
"I think there are a lot of negative messages out there, so it's important for the families to hear the positive message from us."

To address this need, they routinely informed parents that HPV vaccine prevents cancer and sometimes they mentioned the prevention of warts. However, few routinely discussed vaccine safety, preferring to provide this information if parents expressed specific safety concerns. Few used parent educational materials apart from the required vaccine information sheets (VIS) from the CDC. Several providers noted that teenagers often were concerned about getting the HPV vaccine as they had heard it was more painful than other vaccines, and some teens were reluctant to get all three adolescent vaccines at the same visit.

\section{Peer pressure}

A few providers described pressure from a partner in their practice to change their approach to HPV vaccine implementation.

\section{External policy and incentives}

All providers were aware of the national guideline recommendations to complete the 3-dose series by age 13. Many parents chose to defer HPV vaccine as it is not required for school attendance. Few providers were aware of financial incentives including those offered by vaccine manufacturers to increase completion of the 3dose series and by Medicaid to promote meaningful use of clinical information.

\section{Cosmopolitan}

All providers reported that they learned about the recommendations for HPV vaccine from external sources, most commonly through membership with the American Academy of Pediatrics (AAP) list serve. They also received 
information from "throw away" medical journals, drug representatives, and the internet, and were unlikely to seek or receive information about the vaccine from local infectious disease experts.

\section{Inner setting domain \\ Networks and communication}

Except for the solo-practitioner, all providers reported formal or informal opportunities for communication with other providers in their practice, typically through formal meetings or shared office space. However, the majority of providers were unaware of how their colleagues approached delivery of HPV vaccine.

\section{Readiness for implementation}

Few providers reported leadership engagement in system-level improvement activities or use of available resources (such as their EMR and reminder calls) to increase use of HPV vaccine. Only one provider reported training for office staff involved with vaccine delivery.

\section{Implementation climate}

In some practices that delivered both the other adolescent vaccines at the 11-12 year old check-up visit, delivery of HPV vaccine fit well with existing workflows. In others, delivery required additional work at a subsequent check-up visit. More than half of providers reported that increasing use of HPV vaccine was a relatively high priority for improvement within their practice, but there were no statements that demonstrated a tension for change. Although all aspired to $100 \%$ vaccine coverage, none had any formal goals for themselves or their practice, or monitored vaccine coverage.

\section{Characteristics of individuals domain Knowledge and beliefs}

All providers believed that HPV vaccine was an important vaccine and had a personal investment in providing the vaccine. Several routinely provided vaccine-related facts about HPV vaccine to parents such as usage data to address safety concerns. The majority of providers were aware that earlier vaccination resulted in an increased immune response. They varied in their belief that the vaccine should be initiated at age 11-12 years, and that there was benefit to initiating the HPV series by bundling with Tdap and MCV4. While some were very supportive of this "bundling," others thought it was "a harder sell." Several providers were reluctant to immunize pre-pubertal teens and tailored their recommendation to their perception of the child's risk of sexual activity. One provider mentioned concern that the duration of protection may not be adequate if provided at age 11-12.

\section{Self-efficacy}

Providers varied in their confidence to strongly recommend HPV vaccine at the 11-12 year visit and to convince hesitant parents to accept it. Many expressed frustration with hesitant parents as demonstrated by this quote:

"You can talk until you're blue in the face and give every ounce of evidence and research behind what you do and why you do it. They don't listen. It just-it's frustrating as a provider. Extremely frustrating."

\section{Readiness to change}

Several providers had engaged in informal efforts to change their approach to increase vaccine use. An example is described by this provider.

"I used to say, "I have an optional vaccine." And, I found that when I said that, I had a very, very low, um, percentage of patients who agreed to take it. So, I don't use the word 'optional' anymore."

\section{Process domain \\ Planning}

No-one used a formal planning process initially to implement HPV vaccine or subsequently to change their approach to vaccine use.

\section{Engaging}

Few providers had actively engaged staff members in problem solving to increase vaccine use or expected their Medical Assistants (MAs) to endorse vaccination.

“[The MA's] don't really influence the parents' decisions about vaccines... They administer the vaccines, but they're not counseling."

\section{Executing}

Providers universally assumed responsibility for vaccine delivery and reported the same approach for girls and boys. Typically, they recommended the HPV vaccine at the 11-12 year old visit, although the strength of this recommendation varied. Some providers routinely shared personal information about vaccinating their children or grandchildren, while others only provided this information if asked. One provider had the MA present all three vaccines as "the vaccines recommended today," and only discussed benefits and concerns if requested by the parent. He suggested that this MA-driven process of presenting this vaccine as "no different from any other vaccine" was very effective. He thought that the more traditional 
approach of "We're gonna do this vaccine today, this vaccine today, and your doctor will discuss this vaccine with you today. ..." both "justified the concern that families have if they came in with it and it created concern in families that didn't."

Teamwork to deliver the vaccine series was uncommon. Many providers were unaware of the approach their MA took with parents regarding follow-up doses after they had immunized them with the first dose.

All providers reported that they had vaccine-only visits and offered follow-up doses at acute care visits. Few used practice-level strategies to support subsequent doses such as routinely booking follow-up appointments or making reminder calls to parents. Most often the responsibility to schedule the 2nd and 3rd doses was left to the parent.

\section{Reflecting and evaluating}

No providers reviewed HPV vaccine coverage data. One provider commented that, "it's not as easy as you think to get the data out of here [the EMR]."

\section{Integrated data results}

Of the 72 CFIR constructs assessed in the table with the coverage rates, seven strongly distinguished and seven weakly distinguished between providers with higher versus lower HPV vaccine coverage. These constructs are briefly described here and are summarized in Table 4 as facilitators and barriers to vaccine use.

\section{Intervention characteristics domain}

Within the intervention characteristics domain, relative advantage and adaptability of the age of initiation were strongly distinguishing constructs, one acting as a facilitator and one as a barrier to vaccine use. All providers in the higher coverage group had positive ratings for perceiving a relative advantage to providing the vaccine at age 11-12 years as suggested by the guidelines compared to delaying until the teen is older, as characterized in this quote.

"We would recommend the Tdap, the Menactra, as well as the HPV. ...We recommend starting the HPV at that age, simply because the earlier you start it, the more effective it becomes. You want to start it because you want to get the kids before there's any concern for any exposure. You wanna make sure that they complete their series. We always routinely recommend that at $11 . "$

Table 4 Facilitators and Barriers for completing the HPV vaccine series by 13th birthday

\begin{tabular}{|c|c|c|}
\hline CFIR Domain & Facilitators & Barriers \\
\hline $\begin{array}{l}\text { Characteristics of HPV vaccine } \\
\text { recommendations }\end{array}$ & $\begin{array}{l}\text { *Perceived advantage to vaccinate at age 11/12 } \\
\text { compared to older: } \\
\text { - Increased immunogenicity } \\
\text { - Efficient; Can bundle } \\
\text { - Complete series before exposure } \\
\text { - Easier patient access }\end{array}$ & $\begin{array}{l}\text { *Adapt recommendation to initiate } \\
\text { at older age }\end{array}$ \\
\hline External environment and context & $\begin{array}{l}\text { Financial incentives for series completion: } \\
\text { - Increased billing } \\
\text { - Meaningful use for Medicaid }\end{array}$ & $\begin{array}{l}\text { *Not mandated by school therefore } \\
\text { offer as optional at age } 11 / 12\end{array}$ \\
\hline \multirow[t]{3}{*}{ Internal context of practice } & $\begin{array}{l}\text { - Communication across staff and providers to } \\
\text { coordinate series completion }\end{array}$ & \\
\hline & $\begin{array}{l}\text { - Leadership engaged in system level improvements } \\
\text { e.g., EMR }\end{array}$ & \\
\hline & $\begin{array}{l}\text { - Use of multiple available resources e.g., EMR alert, } \\
\text { outreach calls }\end{array}$ & \\
\hline \multirow[t]{5}{*}{ Characteristics and attitudes of providers } & - *Perceive value in completing series by age 13 & \\
\hline & - Perceive value in bundling 3 adolescent vaccines & \\
\hline & $\begin{array}{l}\text { - }{ }^{*} \text { Confident to strongly recommend HPV vaccine } \\
\text { and to convince hesitant parents }\end{array}$ & \\
\hline & • Enthusiastic about HPV vaccine & \\
\hline & - *Already made efforts to increase HPV vaccine use & \\
\hline \multirow[t]{3}{*}{$\begin{array}{l}\text { Process for implementation of HPV vaccine } \\
\text { recommendations }\end{array}$} & $\begin{array}{l}\text { - } \text { Routinely provide strong recommendation for HPV } \\
\text { vaccine at age } 11 / 12 \text { and bundle } 3 \text { vaccines }\end{array}$ & \\
\hline & - Involve staff in meaningful problem-solving & \\
\hline & $\begin{array}{l}\text { - Reflect on HPV vaccine use with a view to making } \\
\text { changes }\end{array}$ & \\
\hline
\end{tabular}


None of the providers with higher coverage routinely adapted the recommended age of vaccine initiation. Providers with lower coverage routinely initiated the vaccine series at a later age when they described the parents as "more accepting."

\section{Outer setting domain}

Within the outer setting domain, patient needs and resources was a strongly distinguishing construct. Providers who anticipated that parents wanted to know which vaccines offered at the 11-12 year old checkup were mandatory for school attendance routinely suggested HPV vaccine as optional at that visit. These providers also had lower vaccine coverage suggesting this construct is a barrier to HPV vaccine use. One provider described her typical approach:

"Because the Menactra is, and the Tdap are required by school, I guess I introduce them by saying these are the vaccines they get at 11 , in order to go to school. The other one I say we highly encourage that your child gets the HPV."

None of the providers with higher coverage adjusted the adolescent vaccine recommendation in this way and they were more likely to routinely discuss vaccine safety than those with lower coverage. Other factors in this domain weakly distinguished between higher and lower vaccine coverage. Providers with higher coverage were less likely to feel peer pressure to change their implementation approach and were more likely to have positive ratings for responding to financial incentives as demonstrated by this quote:

"...... we're imagining that a lot of the parameters they're using for meaningful-use will also be for pay for performance... and so we wanna make sure our percentages are up there so, we're, we're always very cognizant of those red flags (... to indicate a vaccine is due) and, and that they need to be checked off." ... "That, that's the main incentive. Um, you know. Meaningful use."

These findings suggest that a statement of vaccine safety and awareness of financial incentives can facilitate vaccine use.

\section{Inner setting domain}

Within the inner setting domain, patterns of ratings in the dimensions of networks and communications and readiness for implementation weakly distinguished providerlevel vaccine coverage, acting to facilitate use. Providers who communicated with staff to coordinate vaccine use; reported a consistent approach across providers in their practice; described leadership engagement in system-level improvement activities and use of available resources to provide HPV vaccine had higher vaccine coverage than those who did not report these features.

\section{Characteristics of individuals domain}

Within the characteristics of the individual domain, many factors distinguished between higher and lower vaccine coverage, all facilitating vaccine use. The knowledge and beliefs of the providers about HPV vaccine was a strongly distinguishing construct. Providers with higher coverage were more likely to believe there was value in completing the vaccine series by age 13 and in bundling the three recommended adolescent vaccines at the 11-12 year checkup visit. Provider self-efficacy was a strongly distinguishing construct as was readiness to change of individual providers. Providers with higher coverage were more likely to be enthusiastic and confident in strongly recommending the vaccine at age 11-12 and in their ability to convince hesitant parents to accept the vaccine as demonstrated by this quote:

“...I feel like I'm able to work through their concerns, rebut any inaccuracies that they come in with, and give them the right information they need to make a decision to proceed."

In contrast, those with lower coverage did not share this confidence as demonstrated by this quote:

“...typically, if they're not going to give it, nothing you're going to tell them is going to change their mind."

Those with higher coverage were more likely to already have engaged in personal efforts to increase HPV vaccine use such as adding provider reminders to the EMR.

\section{Process domain}

Within the process domain, executing was a strongly distinguishing construct. All providers with higher coverage routinely strongly recommended and routinely provided the vaccine at the 11-12 year old check-up visit, and bundled the three adolescent vaccines. Also, they were more likely to provide personal information about HPV vaccine use as described by one provider:

"I give a quick spiel about how I think it's really important, how it helps protect against cancer, how it's safe, we've given lots of doses, nationwide lots of doses have been given, it's effective, it prevents cancer, I give it to my kids and lots of kids every day, I think it's really important..." 
Several constructs weakly distinguished between providers with higher and lower coverage. Evidence of planning, engaging, and reflecting and evaluating was most often associated with higher coverage, suggesting these constructs facilitate use.

\section{Discussion}

Study findings both confirm that effective strategies to increase HPV vaccine use are urgently needed and inform their development. By systematically examining the complex web of factors that influence use of this vaccine in the primary care setting, we identified important facilitators and barriers to following National guidelines. We learned that a multi-level approach is needed to markedly increase use of this important vaccine. At the providerlevel, buy-in to vaccination by age 13 is critical as is the provider's confidence to address parental hesitancy. At the interpersonal-level, our findings suggest several strategies the provider could use with the patient, parents and staff to increase vaccine use. These include recommending the three adolescent vaccines as a bundle and addressing parental hesitation with a strong and persistent recommendation for timely HPV vaccine use. Better co-ordination between the provider and staff who administer the vaccine could standardize communication messages for patients and parents and ensure opportunities for vaccination are not missed. At the practice-level, collaboration among providers could improve efficiency by standardizing use of reminder systems and maximize benefit from financial incentives.

Our findings confirm that the provider's approach to HPV vaccine personally, interpersonally and at the practice level, strongly influences uptake. $[15,16]$ Providers in these independent community practices universally assumed responsibility for vaccine delivery, and reported they routinely recommended HPV vaccine, Tdap and MCV4 to their patients at their 11-12 year-old checkup visit. Our analysis demonstrated that the quality of their recommendation varied widely as did HPV vaccine coverage by age 13 . These findings are consistent with national studies with larger samples $[17,18]$ and likely reflect the complexity of communication about this vaccine. [18] Failure to require HPV vaccine for school attendance influenced some providers to suggest that the vaccine could be delayed [19] and anticipation of parental resistance caused them to feel compelled to have a conversation about the vaccine. This likely contributed to reduced vaccine uptake as parents are more likely to resist vaccination recommendations if the provider uses a participatory, conversational approach rather than a more directive approach (a presumptive or announcement approach). [20, 21] It is likely that providers' lack of buy-in to vaccination by age 13 and their low self-efficacy to address parental hesitancy further compromised their ability to provide a strong recommendation. At the practice-level, reminder and recall systems that can increase vaccine series initiation and completion, $[9,10,22,23]$ were infrequently used. Many providers in our sample lacked resources such as information technology (IT) support to augment their EMR with these systems. [24]

Effective implementation strategies must help providers to change their approach to HPV vaccine. However, changing provider behavior is difficult. Educational interventions targeting providers have not been successful, [9] and reminders and audit and feedback have had mixed results. [9] Although lack of a strong provider recommendation is reputed to be the most common barrier to use of HPV vaccine, [15-17, 25] few studies have tried to improve the quality of provider communication. One randomized trial conducted in the primary care setting showed that training providers to use an announcement approach increased vaccine coverage by $5 \%$, [8] but the broader literature of message framing has yielded mixed results. [18] In addition to changing provider behavior regarding the timing and quality of their recommendation for HPV vaccine, our findings suggest the need for providers to develop an adolescent vaccine delivery system. While a systematic approach was effective in a large healthcare system with access to staff and technical support for implementation, [19] most interventions to systematize vaccination in primary care practices have been disappointing and expensive. [9] [26] A recent randomized controlled trial showed an intervention comprised of provider education, audit and feedback and EMR reminders increased vaccine initiation by $8 \%$ and the intervention was relatively inexpensive, but may not be scalable without IT support. [27] Effective, scalable interventions to encourage and support providers in independent practices to deliver HPV vaccine to all eligible teens in a timely fashion are needed. [10]

Our findings inform an implementation strategy to change provider behavior regarding this complex vaccine. An acceptable approach will be simple to use, save time, and alleviate the stress associated with dealing with vaccine hesitant parents. [28] Key ingredients might include the following: 1) Education to motivate behavior change and increase self-efficacy. Providers must be convinced of the benefits of early initiation of the vaccine series and able to succinctly and comfortably address parental concerns. 2) To facilitate change, additional pragmatic resources such as a communication strategy to increase the quality of vaccine recommendation are needed. 3) Targeting increased coverage for any age before the 15th birthday may engage more providers in the change process and capitalize on the latest recommendation for a 2-shot series. [5] It would also 
significantly impact the Healthy People 2020 goal of $80 \%$ coverage for 13-15 year olds. 4) Finally, engaging staff in developing and implementing a new vaccine delivery system to increase vaccine use would be beneficial. Failure to simultaneously address all these factors may explain why prior interventions to improve use of HPV vaccine have had at best a modest effect and changes have not been sustained.

Our study is limited by several considerations. Although we believe the study sample is representative of providers working in independent, community-based pediatric practices in our community with considerable variation in the context of where and how the providers practiced, our convenience sample of 16 providers is small and from one geographic area and may not be representative of other populations. Completion of the 3-dose series before the 13th birthday was low among participants, consistent with national data. [7] As no providers had high vaccine uptake, our findings regarding barriers and facilitators for HPV vaccine uptake should be considered as exploratory. The time span for collecting the quantitative and qualitative data were different (2014 and 2016 respectively. National data show the increase in HPV vaccine coverage from 2014 to 2015 was minimal, and suggest that a dramatic change between 2014 and 2016 is unlikely. [7, 29] However, if changes in coverage were large and not equal across providers, then our assessment of barriers and facilitators may be inaccurate. Finally, our qualitative data was retrospective and we did not interview parents or patients.

\section{Conclusions}

In conclusion, study and national data suggest that many teens are not protected against HPV-related cancers prior to exposure to the virus and that widespread efforts to increase HPV vaccine use are urgently needed. Using CFIR to systematically examine the use of this vaccine in independent primary care practices enabled us to identify facilitators and barriers at the provider, interpersonal and practice level that need to be addressed in future efforts to increase vaccine use in such settings. Our findings suggest that implementation strategies that target the provider and help them to address multi-level barriers to $\mathrm{HPV}$ vaccine use merit further investigation.

\footnotetext{
Abbreviations

AAP: American Academy of Pediatrics; ACIP: Advisory Committee on Immunization Practices; CDC: Centers for Disease Control and Prevention; EMR: Electronic Medical Record; HEDIS: Healthcare Effectiveness Data and Information Set; HPV: Human Papillomavirus; IQR: Inter-quartile range; MCV4: Quadrivalent meningococcal vaccine; NCATS: National Center for Advancing Translational Sciences; $\mathrm{NIH}$ : National Institutes of Health; Tdap: Tetanus, diphtheria and pertussis vaccine; U.S.: United States; VIS: Vaccine information sheet; WU PAARC: Washington University Pediatric and Ambulatory Research Consortium
}

\section{Funding}

This study was supported by the Washington University Institute of Clinical and Translational Sciences grant UL1 TR000448 from the National Center for Advancing Translational Sciences (NCATS) of the National Institutes of Health $(\mathrm{NIH})$, with co-funding from St. Louis Children's Hospital. This study's contents are solely the responsibility of the author and do not necessarily represent the official view of NCATS or NIH. The sponsors had no involvement in the design and conduct of the study; collection, management, analysis, and interpretation of the data; preparation, review, or approval of the manuscript; and decision to submit the manuscript for publication.

\section{Availability of data and materials}

Data sharing is not applicable to this article as no datasets were generated or analyzed during the current study.

\section{Author's contributions}

JMG designed the study, conducted interviews, analyzed and interpreted the qualitative and quantitative data, and drafted the manuscript. SD analyzed and interpreted the qualitative data and critically reviewed and edited the manuscript. EW conducted interviews, analyzed and interpreted the qualitative data, and critically reviewed and edited the manuscript. AAL analyzed and interpreted the qualitative data, and critically reviewed and edited the manuscript. KK analyzed and interpreted the qualitative data, and critically reviewed and edited the manuscript. RL designed the study, analyzed and interpreted the qualitative data, and critically reviewed and edited the manuscript. All authors read and approved the final manuscript.

\section{Ethics approval and consent to participate}

This study was approved by the Washington University Human Research Protection Office (Ref \# 210510093). Each participant provided written consent.

\section{Consent for publication}

We confirm that the consent form signed by all participants provides consent to publish anonymous quotes within the manuscript.

\section{Competing interests}

The authors declare that they have no competing interests.

\section{Publisher's Note}

Springer Nature remains neutral with regard to jurisdictional claims in published maps and institutional affiliations.

\section{Author details}

${ }^{1}$ Departments of Medicine and Pediatrics, Washington University School of Medicine, St. Louis, MO, USA. ${ }^{2}$ Department of Pediatrics, Washington University School of Medicine, Campus Box 8116, 660 S. Euclid Ave, St. Louis, MO 63110, USA. ${ }^{3}$ Department of Pediatrics, Michigan Medicine, C.S. Mott Children's Hospital, Ann Arbor, USA. ${ }^{\circ}$ Department of Surgery, Washington University School of Medicine, St. Louis, MO, USA.

Received: 22 August 2017 Accepted: 23 April 2018

Published online: 07 May 2018

\section{References}

1. Fu LY, Bonhomme LA, Cooper SC, Joseph JG, Zimet GD. Educational interventions to increase HPV vaccination acceptance: a systematic review. Vaccine. 2014;32(17):1901-20.

2. President's Cancer Panel Annual Report 2012-2013 [http://deainfo.nci.nih.gov/ advisory/pcp/annualReports/HPV/PDF/PCP_Annual_Report_2012-2013.pdf]. Accessed 30 Apr 2018.

3. Gertig DM, Brotherton JM, Budd AC, Drennan K, Chappell G, Saville AM. Impact of a population-based HPV vaccination program on cervical abnormalities: a data linkage study. BMC Med. 2013;11:227.

4. Markowitz LE, Dunne EF, Saraiya M, Chesson HW, Curtis CR, Gee J, Bocchini JA Jr, Unger ER, Centers for Disease C, Prevention. Human papillomavirus vaccination: recommendations of the Advisory Committee on Immunization Practices (ACIP). MMWR Recomm Rep. 2014;63(RR-05):1-30.

5. Meites $E$, Kempe A, Markowitz LE. Use of a 2-dose schedule for human papillomavirus vaccination - updated recommendations of the advisory 
committee on immunization practices. MMWR Morb Mortal Wkly Rep. 2016;65(49):1405-8.

6. Healthy People 2020 [http://healthypeople.gov/]. Accessed 30 Apr 2018.

7. Reagan-Steiner S, Yankey D, Jeyarajah J, Elam-Evans LD, Curtis CR, MacNeil J, Markowitz LE, Singleton JA. National, regional, state, and selected local area vaccination coverage among adolescents aged 13-17 years - United States, 2015. MMWR Morb Mortal Wkly Rep. 2016;65(33):850-8

8. Brewer NT, Hall ME, Malo TL, Gilkey MB, Quinn B, Lathren C. Announcements Versus Conversations to Improve HPV Vaccination Coverage: A Randomized Trial. Pediatrics. 2017;139(1):e20161764.

9. Smulian EA, Mitchell KR, Stokley S. Interventions to increase HPV vaccination coverage: a systematic review. Human vaccines \& immunotherapeutics. 2016;12(6):1566-88.

10. Niccolai LM, Hansen CE. Practice- and community-based interventions to increase human papillomavirus vaccine coverage: a systematic review. JAMA Pediatr. 2015;169(7):686-92.

11. Damschroder LJ, Aron DC, Keith RE, Kirsh SR, Alexander JA, Lowery JC. Fostering implementation of health services research findings into practice: a consolidated framework for advancing implementation science. Implementation science : IS. 2009;4:50.

12. Fetters MD, Curry LA, Creswell JW. Achieving Integration in Mixed Methods Designs - Principles and Practices. Health Serv Res. 2013;48:1-23.

13. Immunization and Infectious Diseases [https:/www.healthypeople.gov/2020/ topics-objectives/topic/immunization-and-infectious-diseases/objectives]. Accessed 30 Apr 2018.

14. Palinkas LA. Qualitative and mixed methods in mental health services and implementation research. J Clin Child Adolesc Psychol. 2014;43(6):851-61.

15. Holman DM, Benard V, Roland KB, Watson M, Liddon N, Stokley S. Barriers to human papillomavirus vaccination among US adolescents: a systematic review of the literature. JAMA Pediatr. 2014;168(1):76-82.

16. Perkins RB, Clark JA, Apte G, Vercruysse JL, Sumner JJ, Wall-Haas CL, Rosenquist AW, Pierre-Joseph N. Missed opportunities for HPV vaccination in adolescent girls: a qualitative study. Pediatrics. 2014;134(3):e666-74.

17. Gilkey MB, Calo WA, Moss JL, Shah PD, Marciniak MW, Brewer NT. Provider communication and HPV vaccination: the impact of recommendation quality. Vaccine. 2016;34(9):1187-92.

18. Gilkey MB, McRee AL. Provider communication about HPV vaccination: a systematic review. Human vaccines \& immunotherapeutics. 2016; 12(6):1454-68.

19. Farmar AM, Love-Osborne K, Chichester K, Breslin K, Bronkan K, Hambidge SJ. Achieving High Adolescent HPV Vaccination Coverage. Pediatrics. 2016;138(5):e20152653.

20. Opel DJ, Mangione-Smith R, Robinson JD, Heritage J, DeVere V, Salas HS, Zhou C, Taylor JA. The influence of provider communication behaviors on parental vaccine acceptance and visit experience. Am J Public Health. 2015; 105(10):1998-2004.

21. Opel DJ, Heritage J, Taylor JA, Mangione-Smith R, Salas HS, Devere V, Zhou C, Robinson JD. The architecture of provider-parent vaccine discussions at health supervision visits. Pediatrics. 2013;132(6):1037-46.

22. Szilagyi PG, Albertin C, Humiston SG, Rand CM, Schaffer S, Brill H, Stankaitis J, Yoo BK, Blumkin A, Stokley S. A randomized trial of the effect of centralized reminder/recall on immunizations and preventive care visits for adolescents. Acad Pediatr. 2013;13(3):204-13.

23. Tierney CD, Yusuf H, McMahon SR, Rusinak D, OB MA, Massoudi MS, Lieu TA. Adoption of reminder and recall messages for immunizations by pediatricians and public health clinics. Pediatrics. 2003;112(5):1076-82.

24. Wolfson D, Bernabeo E, Leas B, Sofaer S, Pawlson G, Pillittere D. Quality improvement in small office settings: an examination of successful practices. BMC Fam Pract. 2009:10:14.

25. Darden PM, Thompson DM, Roberts JR, Hale JJ, Pope C, Naifeh M, Jacobson RM. Reasons for not vaccinating adolescents: National Immunization Survey of teens, 2008-2010. Pediatrics. 2013;131(4):645-51.

26. Perkins RB, Zisblatt L, Legler A, Trucks E, Hanchate A, Gorin SS. Effectiveness of a provider-focused intervention to improve HPV vaccination rates in boys and girls. Vaccine. 2015;33(9):1223-9.

27. Fiks AG, Grundmeier RW, Mayne S, Song L, Feemster K, Karavite $D$, Hughes CC, Massey J, Keren R, Bell LM, et al. Effectiveness of decision support for families, clinicians, or both on HPV vaccine receipt. Pediatrics. 2013;131(6):1114-24.

28. Kempe A, O'Leary ST, Kennedy A, Crane LA, Allison MA, Beaty BL, Hurley LP, Brtnikova M, Jimenez-Zambrano A, Stokley S. Physician response to parental requests to spread out the recommended vaccine schedule. Pediatrics. 2015;135(4):666-77.

29. Laurie D, Elam-Evans DY, Jeyarajah J, Singleton JA, Robinette Curtis C, MacNeil J, Hariri S. National, Regional, State, and Selected Local Area Vaccination Coverage Among Adolescents Aged 13-17 Years - United States, 2013. In: Morbidity and Mortality Weekly Report (MMWR), vol. 63. Atlanta: Centers for Disease Control and Prevention; 2014. p. 625-33.

\section{Ready to submit your research? Choose BMC and benefit from:}

- fast, convenient online submission

- thorough peer review by experienced researchers in your field

- rapid publication on acceptance

- support for research data, including large and complex data types

- gold Open Access which fosters wider collaboration and increased citations

- maximum visibility for your research: over $100 \mathrm{M}$ website views per year

At BMC, research is always in progress.

Learn more biomedcentral.com/submissions 